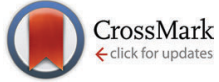

Cite this: Phys. Chem. Chem. Phys., 2015, 17, 5913

Received 6th December 2014, Accepted 23rd January 2015

DOI: $10.1039 / c 4 c p 05704 j$

www.rsc.org/pccp

\title{
Long-lived nuclear spin states far from magnetic equivalence $\dagger$
}

\author{
Gabriele Stevanato, Soumya Singha Roy, Joe Hill-Cousins, Ilya Kuprov, \\ Lynda J. Brown, Richard C. D. Brown, Giuseppe Pileio and Malcolm H. Levitt*
}

\begin{abstract}
Clusters of coupled nuclear spins may form long-lived nuclear spin states, which interact weakly with the environment, compared to ordinary nuclear magnetization. All experimental demonstrations of longlived states have so far involved spin systems which are close to the condition of magnetic equivalence, in which the network of spin-spin couplings is conserved under all pair exchanges of symmetry-related nuclei. We show that the four-spin system of trans-[2,3- $\left.{ }^{13} C_{2}\right]$-but-2-enedioate exhibits a long-lived nuclear spin state, even though this spin system is very far from magnetic equivalence. The 4-spin longlived state is accessed by slightly asymmetric chemical substitutions of the centrosymmetric molecular core. The long-lived state is a consequence of the locally centrosymmetric molecular geometry for the trans isomer, and is absent for the cis isomer. A general group theoretical description of long-lived states is presented. It is shown that the symmetries of coherent and incoherent interactions are both important for the existence of long-lived states.
\end{abstract}

\section{Introduction}

Clusters of coupled nuclear spins may form long-lived states (LLS) with decay time constants $T_{\mathrm{LLS}}$ much longer than the conventional spin-lattice relaxation time $T_{1} \cdot{ }^{1-22}$ Such long-lived states have been used to enhance the study of slow processes such as diffusion and flow by NMR, and to enhance the contrast in ligand binding investigations. ${ }^{21,22}$ Long-lived states hold particular promise for extending the range of nuclear hyperpolarization experiments, ${ }^{23-28}$ in which the small $\left(\sim 10^{-5}\right)$ thermal polarization of nuclear spins is temporarily enhanced by many orders of magnitude. In the case of spin systems containing only two nuclear spins-1/2, the long-lived state is known as singlet order. ${ }^{10}$ This represents the difference in population between the singlet state of the spin-1/2 pair, which is antisymmetric with respect to spin exchange, and the mean population of the triplet states, which are symmetric with respect to exchange. Singlet order is immune to intra-pair dipolar relaxation, which is often the dominant mechanism for the $T_{1}$ process. Singlet order lifetimes $T_{\text {LLS }}$ exceeding $\sim 60 T_{1}$ have been reported. ${ }^{13}$ Long-lived singlet order may also be observed for spin-1/2 isotopes of different type (heteronuclear singlet order). ${ }^{29}$

In systems of more than two coupled spins-1/2, the existence of long-lived singlet order (and LLS in general) depends strongly on

School of Chemistry, University of Southampton, University Road, Southampton, UK.E-mail:mhl@soton.ac.uk

$\dagger$ Electronic supplementary information (ESI) available. See DOI: 10.1039/ c4cp05704j the geometrical arrangement of the nuclei, and the relative magnitudes of spin-spin couplings and chemical shift differences. This problem was examined both theoretically ${ }^{14}$ and numerically. ${ }^{30}$ Multiple-spin states that are protected against intramolecular relaxation mechanisms were predicted to exist, in the case that the rigid geometrical arrangement of nuclei displays local inversion symmetry. ${ }^{30}$ However, no experimental demonstrations of such geometrically imposed long-lived states were provided.

The absence of local geometrical centrosymmetry does not preclude the existence of long-lived spin orders when either the geometrical remoteness of the central spin pair from the other members of the spin system is provided, ${ }^{16-19,25-27}$ or in the presence of fast intramolecular dynamics. ${ }^{31}$

In the following discussion, we provide a clear experimental demonstration of a geometrically-imposed long-lived nuclear spin state in an asymmetrically substituted derivative of ${ }^{13} \mathrm{C}_{2}$-fumarate (trans- $\left[2,3{ }^{13} \mathrm{C}_{2}\right]$-but-2-enedioate). As discussed below, this system exhibits local centrosymmetry but is far from the regime of magnetic equivalence. The confirmed existence of a long-lived state in this system verifies the analyses in ref. 14 and 30 , and shows that near-magnetic-equivalence is not a necessary condition for generating and observing long-lived nuclear spin states.

A theoretical framework accounting for the symmetry properties of the coherent and fluctuating terms in the hamiltonian is introduced for predicting the existence of long-lived spin order. This theory emphasizes the interlocking symmetries of both the coherent and fluctuating parts of the nuclear spin Hamiltonian, as opposed to recent work which only takes the coherent spin interactions into account. ${ }^{16-19}$ 


\section{Near magnetic equivalence}

Consider a pair of nuclei denoted $I_{j}$ and $I_{k}$, with chemical shifts $\delta_{j}$ and $\delta_{k}$, so that their chemical shift frequencies in a magnetic field $B^{0}$ are given by $\omega_{j}^{0}=-\gamma_{j} B^{0}\left(1+\delta_{j}\right)$ where $\gamma_{j}$ is the magnetogyric ratio of $I_{j}$, and similarly for $I_{k}$. Their mutual $J$-coupling is denoted $J_{j k}$. The nuclei are considered to be chemically equivalent if the symmetry of the molecule ensures equal chemical shifts $\delta_{j}=\delta_{k}$. If there are no other nuclei in the system, chemical equivalence also implies magnetic equivalence. However, if there are other nuclei $\left\{I_{l} \ldots\right\}$ in the system, with finite couplings to one or both of $I_{j}$ and $I_{k}$, then a chemically-equivalent pair of nuclei is only magnetically equivalent if all out-of-pair couplings are equal, i.e. $J_{j l}=J_{k l} \forall l \notin\{j, k\} .{ }^{32}$

We define a pair of nuclei $I_{j}$ and $I_{k}$ to exhibit near magnetic equivalence if the following condition holds:

$$
\left(\omega_{j}^{0}-\omega_{k}^{0}\right)^{2}+\pi^{2}\left(J_{j l}-J_{k l}\right)^{2} \leq \pi^{2} J_{j k}^{2} \forall l \notin\{j, k\}
$$

Under conditions of near magnetic equivalence, the mixing of singlet and triplet states by the symmetry-breaking interactions is strongly suppressed by the intra-pair $J$-coupling - a phenomenon called $J$-stabilization. ${ }^{5}$ In this regime, efficient decay of singlet order between spins $I_{j}$ and $I_{k}$ is induced neither by correlated fluctuations of local magnetic fields at the sites of the two spins, nor by fluctuations of the mutual dipole-dipole coupling of $I_{j}$ and $I_{k}$. The suppression of these important relaxation mechanisms often leads to a long-lived state with a relaxation time much longer than the conventional magnetization relaxation time, $T_{1}{ }^{5}$

The condition (1) is depicted graphically in Fig. 1. The horizontal axis indicates the difference in chemically shifted resonance frequencies of the two spins in the pair, while the vertical axis represents the difference of out-of-pair $J$-couplings to other spins in the same molecule. The dashed circle indicates the intra-pair $J$-coupling. Spin pairs may be characterized as being chemically equivalent (corresponding to points on the vertical axis) or magnetically equivalent (point at the origin). Spin pairs with parameters within the dashed circle present near magnetic equivalence.

There have been extensive investigations of isolated spin-1/2 pairs with slightly different chemical shifts, represented by the filled square in Fig. 1. In some cases, the chemical shift difference is sufficiently small that the near-magneticequivalence condition (1) is satisfied without intervention in high magnetic fields. ${ }^{9,12}$ In other cases is satisfied by transporting the sample into a region of low applied magnetic field ${ }^{6,8}$ or by applying resonant radiofrequency fields. ${ }^{33}$ In the regime of near magnetic equivalence, long-lived order may be accessed by carefully timed pulse trains ${ }^{9,34}$ or by continuous radiofrequency fields of suitable amplitude. ${ }^{20,35}$ Appropriate modifications of the terms $\omega_{j}^{0}$ and $\omega_{k}^{0}$ in eqn (1) should be used in these cases.

It is also possible to attain near-magnetic-equivalence by a deviation in the vertical direction, corresponding to small differences in $J$-couplings to spins outside the pair, providing that eqn (1) is still satisfied. This has been demonstrated for chemically equivalent systems (points along the vertical axis). ${ }^{16-19}$

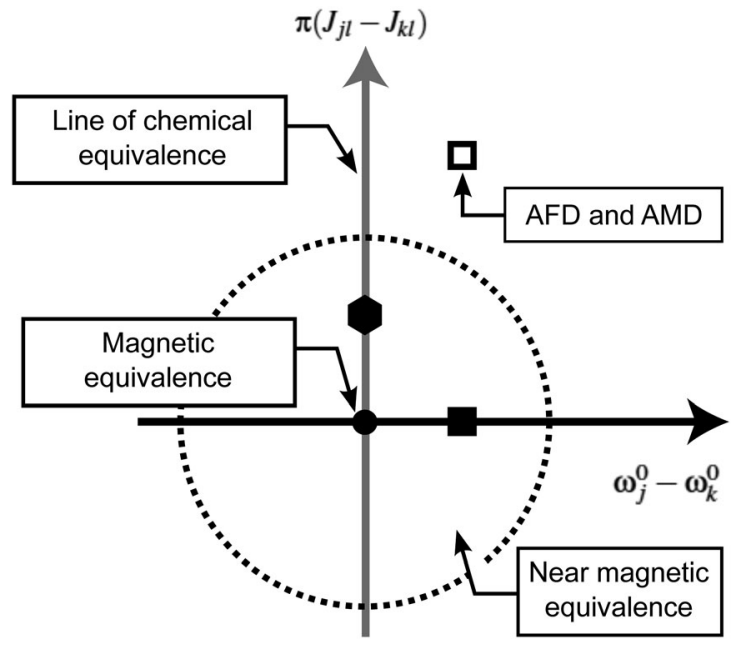

Fig. 1 Pictorial representation of the concepts of chemical and magnetic equivalence for a pair of spins labelled $j$ and $k$. The label $l$ identifies homonuclear spin species coupled with the pair. The dashed circle has a radius $\left|\pi J_{j k}\right|$. The filled circle indicates the point of exact magnetic equivalence. Points on the vertical axis (gray) correspond to systems whose nuclei are chemically equivalent. The filled hexagon represents a spin system whose nuclei are chemically equivalent but slightly magnetically inequivalent. ${ }^{16-19}$ The filled square indicates a spin system formed by two nuclei which are slightly chemically inequivalent. 9,11 The open square, falling well outside the dashed circle, represents spin systems in the regime of strong magnetic inequivalence, as studied in this work.

In this publication we demonstrate the existence of longlived order in a four-spin-1/2 system which does not conform to condition (1). This spin system displays strong magnetic inequivalence with parameters represented by an open square falling well outside the dashed circle in Fig. 1. By comparing two strongly magnetic inequivalent isomers, one exhibiting local centrosymmetry of a four-spin system, and one without centrosymmetry, we show that only the centrosymmetric system displays a long-lived state. In this special case, the long-lived state is geometrically imposed by the local centrosymmetry of the rigid molecular structure, as suggested in ref. 14 and 30. These cases highlight the important role of symmetry in both the coherent and fluctuating spin Hamiltonian, in determining the existence of LLS.

\section{Experimental}

\subsection{Samples}

The molecular structures of the samples used in this study are shown in Fig. 2. Both materials are di-esters of $\left[2,3{ }^{13} \mathrm{C}_{2}\right]$-but-2enedioate which contains a central spin system comprising two ${ }^{13} \mathrm{C}$ nuclei and two ${ }^{1} \mathrm{H}$ nuclei; the fumarate derivative (panels (a) and (c)) has a trans double bond, while the maleate derivative (panes (b) and (d)) has a cis double bond. In both cases, the two ester groups are different, causing the two ${ }^{13} \mathrm{C}$ sites (and their attached protons) to have slightly different chemical shifts. As discussed below, this slight chemical asymmetry is necessary for accessing the long-lived state. All ester groups are deuterated in order to reduce their relaxation contributions. 
a<smiles>[R]OC(=O)C=CC(=O)O[R]</smiles>

b

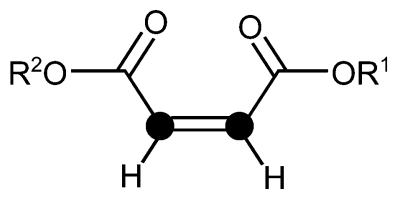

C

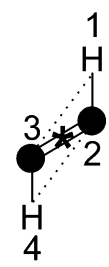

d

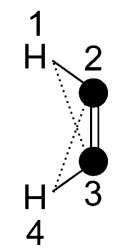

Fig. 2 Molecular structure of (a) ${ }^{13} \mathrm{C}_{2}-\mathrm{AFD}$ and (b) of ${ }^{13} \mathrm{C}_{2}-\mathrm{AMD}$. Filled circles denote ${ }^{13} \mathrm{C}$ nuclei. For both molecules $R^{1}=\mathrm{CD}_{2} \mathrm{CD}_{3}$ and $R^{2}=\mathrm{CD}_{2} \mathrm{CD}_{2} \mathrm{CD}_{3}$. (c) The local spin system of ${ }^{13} \mathrm{C}_{2}-\mathrm{AFD}$, with geometrical inversion centre marked by an asterisk. (d) The local spin system of ${ }^{13} \mathrm{C}_{2}$-AMD.

In the following discussion, the $\left[2,3-{ }^{13} \mathrm{C}_{2}\right]$-fumarate derivative in Fig. 2a [1-(ethyl-d5) 4-(propyl-d7)(E)-but-2-enedioate$2,3-{ }^{13} \mathrm{C}_{2}$ ] is referred to as ${ }^{13} \mathrm{C}_{2}$-AFD (asymmetric fumarate diester); the $\left[2,3-{ }^{13} \mathrm{C}_{2}\right]$-maleate derivative in Fig. $2 \mathrm{~b}$ [1-(ethyl-d5) 4-(propyl-d7)(Z)-but-2-enedioate- ${ }^{13} \mathrm{C}_{2}$ ] is referred to as ${ }^{13} \mathrm{C}_{2}$-AMD (asymmetric maleate diester).

${ }^{13} \mathrm{C}_{2}$-AFD contains a 4 -spin- $1 / 2$ system displaying a local centre of inversion, midway between the two ${ }^{13} \mathrm{C}$ nuclei (Fig. 2c); the local 4-spin-1/2 system in ${ }^{13} \mathrm{C}_{2}$-AMD, on the other hand, displays a reflection plane but is not locally centrosymmetric (Fig. 2d).

The samples were synthesized as described in the ESI. $\dagger$ In both cases $30 \mathrm{mg}$ of the diester were dissolved in $0.5 \mathrm{ml}$ of $\mathrm{CD}_{3} \mathrm{OD}$, added to a Wilmad low pressure/vacuum $5 \mathrm{~mm} \mathrm{NMR}$ tube, degassed thoroughly using the freeze and pump technique ( 5 cycles), and hermetically sealed.

\subsection{NMR experiments}

All NMR experiments were performed at a magnetic field of $11.75 \mathrm{~T}$ on a Bruker $500 \mathrm{MHz}$ Avance III spectrometer using a Bruker $5 \mathrm{~mm}$ triple-resonance liquid-state probe.

The ${ }^{13} \mathrm{C}$ and ${ }^{1} \mathrm{H}$ 1D-NMR spectra were fitted using Spinach simulation software (Fig. 3 and Fig. 4). ${ }^{36,37}$ The obtained chemical shift and coupling parameters are given in Table 1.

The spin systems in both ${ }^{13} \mathrm{C}_{2}$-AMD and ${ }^{13} \mathrm{C}_{2}$-AFD are far from magnetic equivalence. The coupling difference $\left|J_{12}-J_{13}\right|$ is more than twice $\left|J_{23}\right|$, well outside the near-magneticequivalence condition expressed in eqn (1).

Experimental investigation of the long-lived states in the fourspin-1/2 systems of ${ }^{13} \mathrm{C}_{2}$-AFD and ${ }^{13} \mathrm{C}_{2}$-AMD was accomplished by the double-resonance pulse sequences shown in Fig. 5. These pulse sequences are based on the magnetization-to-singlet (M2S) and singlet-to-magnetization (S2M) pulse sequences used to study LLS in homonuclear 2-spin-1/2 systems ${ }^{8,9}$ and in near-equivalent heteronuclear systems. ${ }^{16-19}$ In the current case, each of the M2S
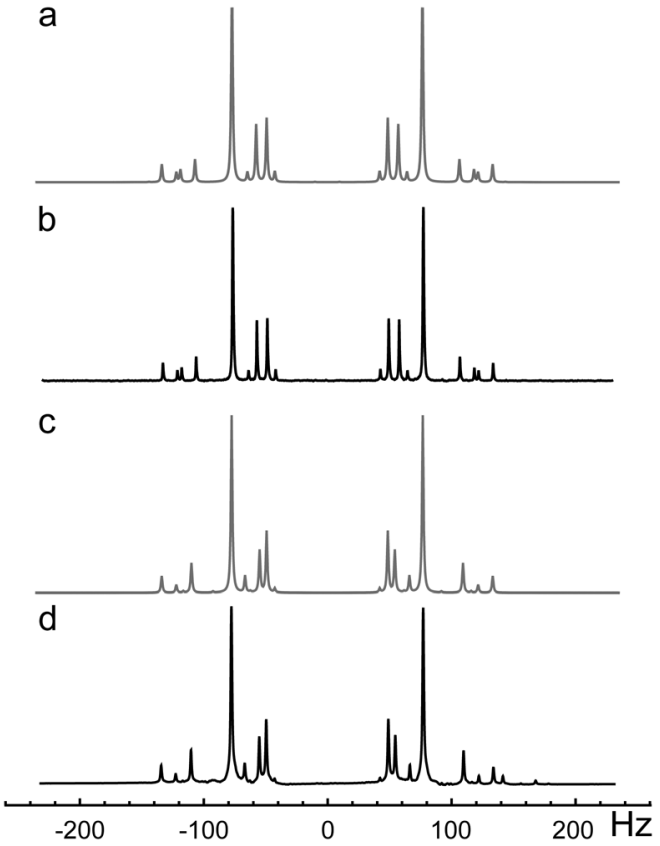

Fig. 3 NMR $1 \mathrm{D}$-spectra of ${ }^{13} \mathrm{C}_{2}$-AFD: (a) simulated ${ }^{13} \mathrm{C}$ spectrum; (b) experimental ${ }^{13} \mathrm{C}$ spectrum. (c) Simulated ${ }^{1} \mathrm{H}$ spectrum; (d) experimental ${ }^{1} \mathrm{H}$ spectrum.

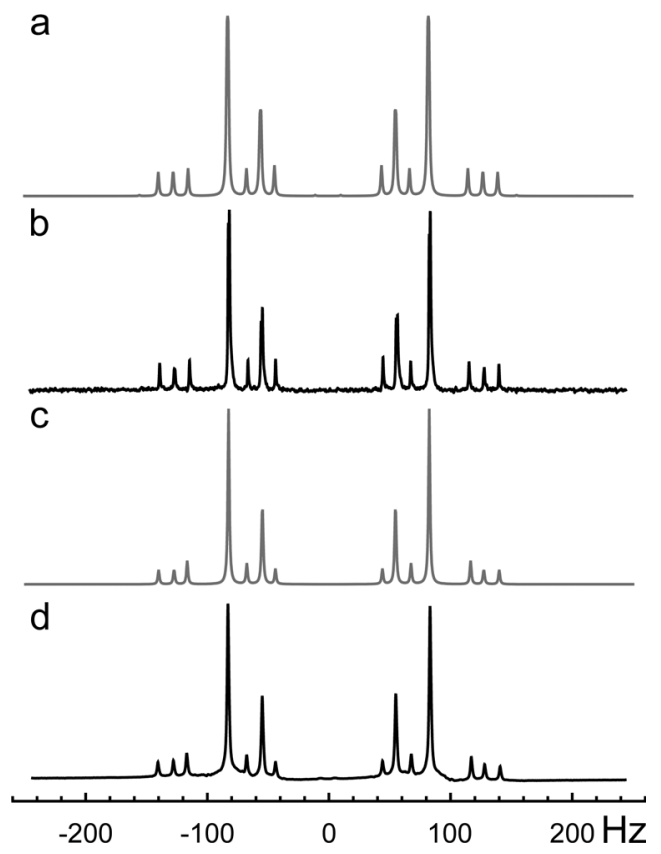

Fig. 4 NMR $1 \mathrm{D}$-spectra of ${ }^{13} \mathrm{C}_{2}$-AMD: (a) simulated ${ }^{13} \mathrm{C}$ spectrum; (b) experimental ${ }^{13} \mathrm{C}$ spectrum. (c) simulated ${ }^{1} \mathrm{H}$ spectrum; (d) experimental ${ }^{1} \mathrm{H}$ spectrum.

and S2M sequences may be performed on either the ${ }^{13} \mathrm{C}$ or the ${ }^{1} \mathrm{H}$ channel, giving rise to the four pulse sequence combinations reported in Fig. 5.

All four sequence variants access the same collective state denoted $\Delta_{\mathrm{gu}}$, as reported by the simulation in Fig. 6 performed using SpinDynamica. ${ }^{39}$ This operator represents the population 
Table 1 Spin system and pulse sequences parameters for ${ }^{13} \mathrm{C}_{2}$-AFD and ${ }^{13} \mathrm{C}_{2}$-AMD in $\mathrm{CD}_{3} \mathrm{OD}$. $\mathrm{J}$ couplings and chemical shift differences were obtained by fitting the 1D-NMR spectra with Spinach. ${ }^{36,37}$ The pulse sequence parameters $\Delta\left({ }^{13} \mathrm{C}\right), \quad \Delta\left({ }^{1} \mathrm{H}\right), \quad n_{1}\left({ }^{13} \mathrm{C}\right), \quad n_{1}\left({ }^{1} \mathrm{H}\right), \quad \omega_{\mathrm{ev}}^{\text {nut }}\left({ }^{13} \mathrm{C}\right) / 2 \pi, \quad \omega_{\mathrm{ev}}^{\text {nut }}\left({ }^{1} \mathrm{H}\right) / 2 \pi$ were optimized experimentally for both molecules. Relaxation time constants $T_{1}$ were measured by standard inversion-recovery at a magnetic field of $11.75 \mathrm{~T}$

\begin{tabular}{llll}
\hline & & ${ }^{13} \mathrm{C}_{2}-\mathrm{AFD}$ & ${ }^{13} \mathrm{C}_{2}-\mathrm{AMD}$ \\
\hline Simulation & $J_{23}$ & $71.0 \pm 0.6 \mathrm{~Hz}$ & $71.6 \pm 0.8 \mathrm{~Hz}$ \\
& $J_{13}=J_{24}$ & $-2.8 \pm 0.03 \mathrm{~Hz}$ & $-1.16 \pm 0.02 \mathrm{~Hz}$ \\
& $J_{12}=J_{34}$ & $166.7 \pm 0.2 \mathrm{~Hz}$ & $166.5 \pm 0.2 \mathrm{~Hz}$ \\
& $J_{14}$ & $15.7 \pm 0.2 \mathrm{~Hz}$ & $11.95 \pm 0.4 \mathrm{~Hz}$ \\
& $\Delta \delta_{23}$ & $62 \pm 5 \mathrm{ppb}$ & $92 \pm 4 \mathrm{ppb}$ \\
& $\Delta \delta_{14}$ & $7 \pm 0.9 \mathrm{ppb}$ & $1.8 \pm 0.2 \mathrm{ppb}$ \\
Experiment & $\Delta\left({ }^{13} \mathrm{C}\right)$ & $3.5 \mathrm{~ms}$ & $3.7 \mathrm{~ms}$ \\
& $\Delta\left({ }^{1} \mathrm{H}\right)$ & $16 \mathrm{~ms}$ & $21 \mathrm{~ms}$ \\
& $n_{1}\left({ }^{13} \mathrm{C}\right)$ & 14 & 14 \\
& $n_{2}\left({ }^{13} \mathrm{C}\right)$ & 7 & 7 \\
& $n_{1}\left({ }^{1} \mathrm{H}\right)$ & 6 & 9 \\
& $n_{2}\left({ }^{1} \mathrm{H}\right)$ & 3 & $520 \mathrm{~Hz}$ \\
& $\omega_{\mathrm{ev}}^{\text {nut }}\left({ }^{1} \mathrm{H}\right) / 2 \pi$ & $520 \mathrm{~Hz}$ & $700 \mathrm{~Hz}$ \\
& $\omega_{\mathrm{ev}}^{\text {nut }}\left({ }^{13} \mathrm{C}\right) / 2 \pi$ & $700 \mathrm{~Hz}$ & $6.0 \pm 0.1 \mathrm{~s}$ \\
& $T_{1}\left({ }^{13} \mathrm{C}\right)$ & $6.0 \pm 0.1 \mathrm{~s}$ & $4.5 \pm 0.1 \mathrm{~s}$ \\
& $T_{1}\left({ }^{1} \mathrm{H}\right)$ & $6.0 \pm 0.1 \mathrm{~s}$ &
\end{tabular}

difference between spin states that are symmetric $(\mathrm{g})$ and antisymmetric $(\mathrm{u})$ with respect to simultaneous exchanges of the ${ }^{1} \mathrm{H}$ and the ${ }^{13} \mathrm{C}$ nuclei, as described below. In the case of ${ }^{13} \mathrm{C}_{2}$-AFD this four-spin state is long-lived. In all cases, the M2S sequence converts either ${ }^{1} \mathrm{H}$ or ${ }^{13} \mathrm{C}$ magnetization into $\Delta_{\text {gu }}$. This state evolves during a variable evolution interval $\tau_{\mathrm{ev}}$, during which an unmodulated irradiation field $(\sim 800 \mu \mathrm{W})$ is applied on both RF channels (nutation frequencies reported in Table 1). This suppresses the small chemical shift difference induced by the different ester substituents. ${ }^{33}$ An S2M sequence, applied on either the ${ }^{1} \mathrm{H}$ or ${ }^{13} \mathrm{C}$ channel, converts the state $\Delta_{\text {gu }}$ into observable transverse magnetization, which is then detected.

A WALTZ-16 decoupling sequence ${ }^{38}$ is applied on the ${ }^{1} \mathrm{H}$ channel during ${ }^{13} \mathrm{C}$ observation, to collapse the spectrum into a single peak. Conversely, the ${ }^{1} \mathrm{H}$ NMR signal is detected in the presence of ${ }^{13} \mathrm{C}$ WALTZ-16 decoupling.

The signal amplitudes for the two compounds, using the four different pulse sequence variants, are plotted against $\tau_{\mathrm{ev}}$ in Fig. 7. The decay time constants reported in Table 2 are consistent among the four different experiments. The experimental points were fitted using a single exponential decay curve when exciting and detecting the spin system on different RF channels. A double exponential decay curve was instead applied when excitation and detection occured on the same RF channel. Further details and the fitting functions are reported in the ESI. $\dagger$ In the case of ${ }^{13} \mathrm{C}_{2}$-AFD, the state $\Delta_{\text {gu }}$ is found to have a decay time constant of $\sim 10 T_{1}$, marking the existence of a four-spin LLS protected against dipole-dipole relaxation and the other mechanisms contributing to the rapid decay of longitudinal magnetization. In the case of ${ }^{13} \mathrm{C}_{2}$-AMD, where a local centre of inversion is absent, the state $\Delta_{\text {gu }}$ decays faster than $T_{1}$ and hence cannot be designated as a long-lived state. The experimental results confirm the link between local molecular geometry and the existence of LLS in multi-spin systems.
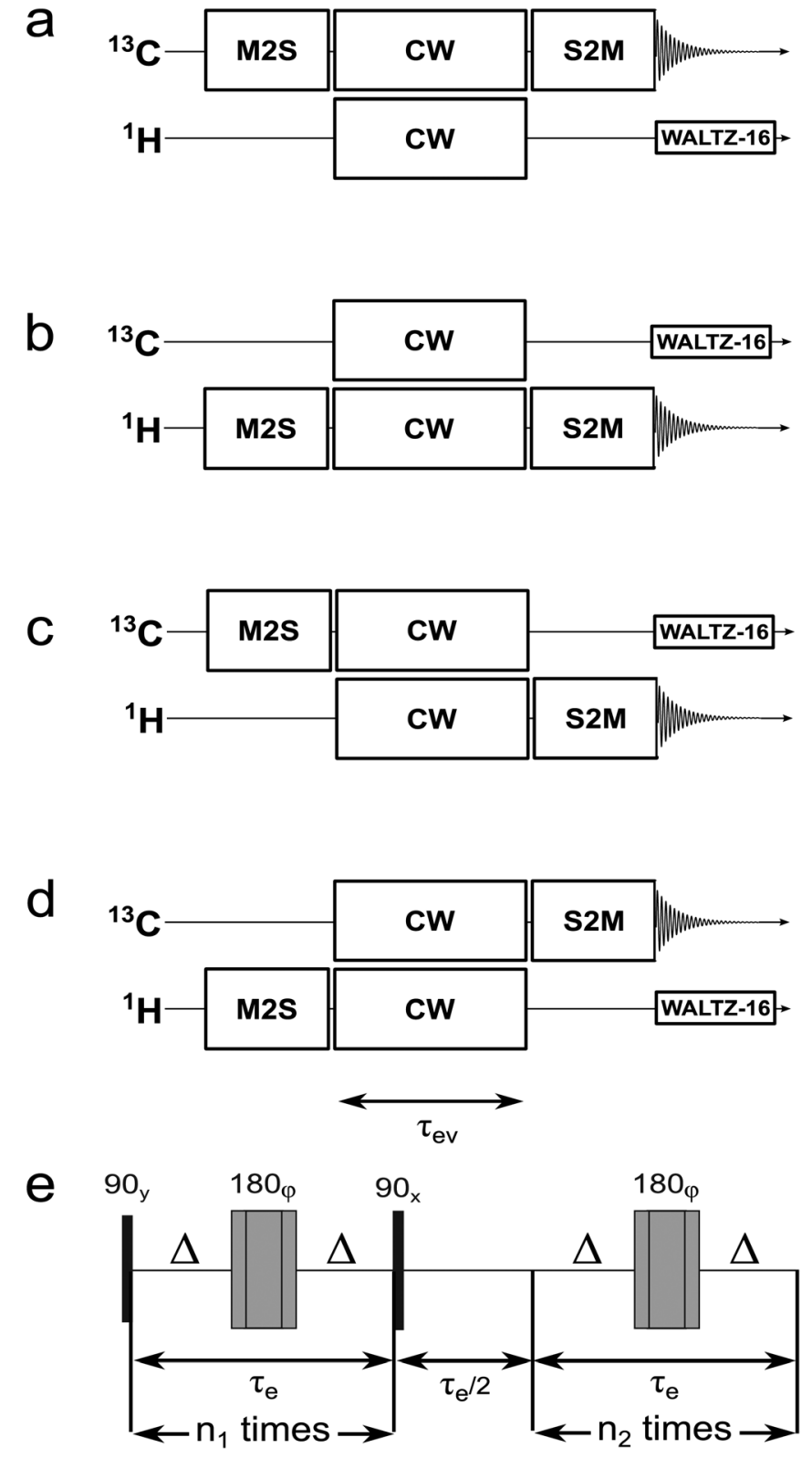

Fig. 5 Pulse sequences used to access the $\Delta_{\text {gu }}$ state in ${ }^{13} \mathrm{C}_{2}$-AFD and ${ }^{13} \mathrm{C}_{2^{-}}$ AMD. Excitation and detection of $\Delta_{\text {gu }}$ is done using M2S and S2M pulse sequences, respectively. M2S and S2M are run on the same RF channel in (a) and (b) or on different channels in (c) and (d). The exact scheme of an M2S sequence is shown in (e). The S2M pulse sequence is obtained by running M2S in reverse time order. The echo duration is given by $\tau_{\mathrm{e}} \sim 1 /\left(2 J_{14}\right)$ when the sequence is applied to the ${ }^{1} \mathrm{H}$ channel, and $\tau_{\mathrm{e}} \sim 1 /\left(2 J_{23}\right)$ when using the ${ }^{13} \mathrm{C}$ channel. The number of loops is always $n_{1}=2 n_{2}$. During the variable evolution time $\tau_{\mathrm{ev}}$ a weak continuous wave irradiation of $800 \mu \mathrm{W}$ is applied on both RF channels $\left(\omega_{\mathrm{ev}}^{\text {nut }}\left({ }^{13} \mathrm{C}\right) / 2 \pi=700 \mathrm{~Hz}, \omega_{\mathrm{ev}}^{\text {nut }}\left({ }^{1} \mathrm{H}\right) / 2 \pi=520 \mathrm{~Hz}\right)$ to impose chemical equivalence. A WALTZ-16 decoupling sequence ${ }^{38}$ is applied to the ${ }^{1} \mathrm{H}$ channel during detection of the ${ }^{13} \mathrm{C}$ signal; WALTZ-16 is applied to the ${ }^{13} \mathrm{C}$ channel when the ${ }^{1} \mathrm{H}$ signal is detected.

\section{Theory}

A nuclear spin system experiences a spin Hamiltonian containing coherent and fluctuating contributions:

$$
\mathcal{H}=\mathcal{H}_{\text {coh }}+\mathcal{H}_{\text {fluc }}(t)
$$




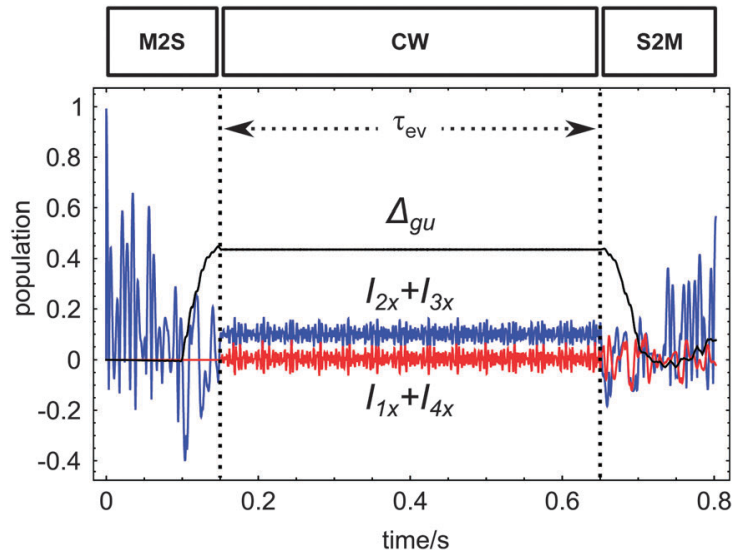

Fig. 6 Simulated trajectories for the transfer of longitudinal magnetization into carbon transverse magnetization $I_{2 x}+I_{3 x}$ in blue, proton transverse magnetization $I_{1 x}+I_{4 x}$ in red and $\Delta_{\text {gu }}$ spin order in black under the pulse sequence in Fig. $5 a$ for ${ }^{13} C_{2}$-AFD. The spin system is assumed to evolve under the influence of $\mathcal{H}_{\text {coh }}$ with parameters reported in Table 1 and $\tau_{\mathrm{ev}}=0.5 \mathrm{~s}$. No relaxation was included in the simulations.

The coherent terms are time-independent and uniform over the ensemble, whereas the fluctuating terms are time-dependent and have different instantaneous values for different ensemble members. In general $\mathcal{H}_{\text {coh }}$ is associated with the frequencies and amplitudes of the NMR peaks, while $\mathcal{H}_{\text {fluc }}$ leads to relaxation. In solution NMR, the symmetry of $\mathcal{H}_{\text {coh }}$ is associated with chemical and magnetic equivalence; the symmetry of $\mathcal{H}_{\text {fluc }}$ is responsible for the existence of long-lived forms of spin order. The existence of LLS in near magnetic equivalent multi-spin systems has been recently analysed in terms of the symmetry of the coherent Hamiltonian alone. ${ }^{17,19}$ As shown below, this can be misleading. The interplay of the various symmetries is now described. Although the discussion will be conducted for the specific cases described in this paper, the general principles may be used to analyze a wide variety of molecular geometries.

\subsection{Symmetry of the coherent hamiltonian}

We write the coherent Hamiltonian as follows:

$$
\mathcal{H}_{\mathrm{coh}}=\mathcal{H}_{\mathrm{coh}}^{0}+\mathcal{H}_{\mathrm{coh}}^{\prime}
$$

where $\mathcal{H}_{\text {coh }}^{0}$ respects the idealized symmetry of the molecular spin system and $\mathcal{H}_{\text {coh }}^{\prime}$ is a small perturbation due to the asymmetric remote substituents. In isotropic liquids, each of these is a superposition of Zeeman and $J$-coupling terms, i.e. $\mathcal{H}_{Z}=\mathcal{H}_{Z}^{0}+\mathcal{H}_{Z}^{\prime}$ and $\mathcal{H}_{J}=\mathcal{H}_{J}^{0}+\mathcal{H}_{J}^{\prime}$, where in the current cases

$$
\begin{gathered}
\mathcal{H}_{Z}^{0}=\frac{1}{2} \omega_{\mathrm{H}}^{0}\left(\delta_{1}+\delta_{4}\right)\left(I_{1 z}+I_{4 z}\right)+\frac{1}{2} \omega_{\mathrm{C}}^{0}\left(\delta_{2}+\delta_{3}\right)\left(I_{2 z}+I_{3 z}\right) \\
\mathcal{H}_{J}^{0}=\pi\left(J_{12}+J_{34}\right)\left(I_{1 z} I_{2 z}+I_{3 z} I_{4 z}\right) \\
+\pi\left(J_{13}+J_{24}\right)\left(I_{1 z} I_{3 z}+I_{2 z} I_{4 z}\right) \\
+2 \pi\left(J_{14} \mathbf{I}_{1} \cdot \mathbf{I}_{4}+J_{23} \mathbf{I}_{2} \cdot \mathbf{I}_{3}\right)
\end{gathered}
$$
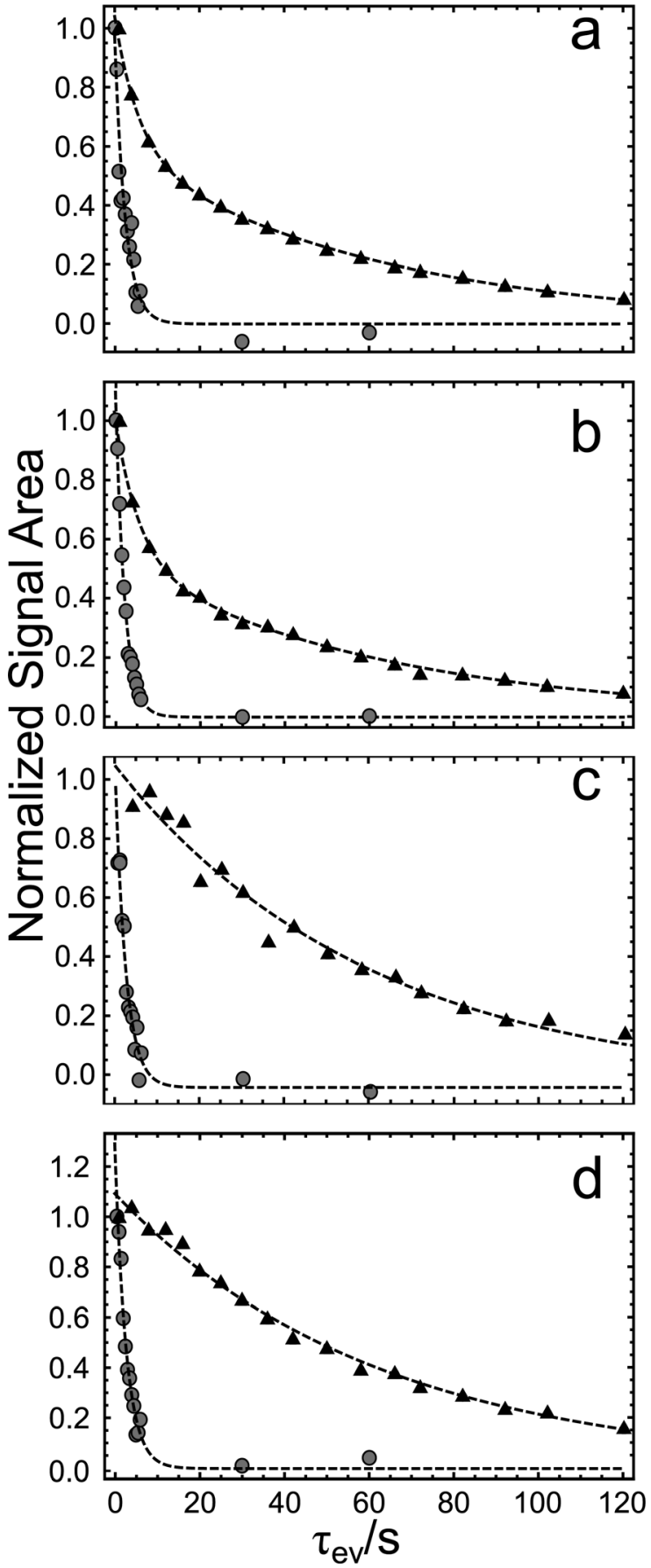

Fig. 7 Experimental results are shown for pulse sequence in (a) Fig. 5a, (b) Fig. 5b, (c) Fig. $5 c$ and (d) Fig. 5 d. Black triangles refer to ${ }^{13} C_{2}-A F D$, and gray circles to ${ }^{13} \mathrm{C}_{2}$-AMD. The dashed lines are best fits to bi-exponential decays in (a) and (b) and single exponential decays in (c) and (d). The time constants for ${ }^{13} \mathrm{C}_{2}$-AFD are: (a) $61.01 \pm 0.1 \mathrm{~s}$; (b) $63.2 \pm 2.5 \mathrm{~s}$; (c) $59.9 \pm 3.2 \mathrm{~s}$; (d) $61.6 \pm 2.0 \mathrm{~s}$. The time constants for ${ }^{13} \mathrm{C}_{2}$-AMD are: (a) $2.5 \pm 0.2 \mathrm{~s}$; (b) 2.1 $\pm 0.1 \mathrm{~s}$; (c) $2.5 \pm 0.2 \mathrm{~s}$; (d) $2.7 \pm 0.2 \mathrm{~s}$.

$$
\begin{aligned}
\mathcal{H}_{j}^{\prime}= & \pi\left(J_{12}-J_{34}\right)\left(I_{1 z} I_{2 z}-I_{3 z} I_{4 z}\right) \\
& +\pi\left(J_{13}-J_{24}\right)\left(I_{1 z} I_{3 z}-I_{2 z} I_{4 z}\right)
\end{aligned}
$$

Here $\omega_{\mathrm{C}}^{0}$ and $\omega_{\mathrm{H}}^{0}$ are the Larmor frequencies of ${ }^{13} \mathrm{C}$ and ${ }^{1} \mathrm{H}$, and $\delta_{i}$ represents the chemical shift of nucleus $I_{i}$. The terms $\mathcal{H}_{Z}^{\prime}$ and 
Table 2 Experimental decay time constants for the spin operator term $\Delta_{\text {gu }}$ as determined by fitting the experimental results in Fig. 7. In the case of ${ }^{13} \mathrm{C}_{2}$-AFD, the operator $\Delta_{\text {gu }}$ is a LLS

\begin{tabular}{|c|c|c|}
\hline Pulse sequence & ${ }^{13} \mathrm{C}_{2}-\mathrm{AFD} / \mathrm{s}$ & ${ }^{13} \mathrm{C}_{2}-\mathrm{AMD} / \mathrm{s}$ \\
\hline $\mathrm{M} 2 \mathrm{~S}\left({ }^{13} \mathrm{C}\right)-\mathrm{S} 2 \mathrm{M}\left({ }^{13} \mathrm{C}\right)$ & 61.01 .0 & $2.5 \quad 0.2$ \\
\hline $\mathrm{M} 2 \mathrm{~S}\left({ }^{1} \mathrm{H}\right)-\mathrm{S} 2 \mathrm{M}\left({ }^{1} \mathrm{H}\right)$ & $63.2 \quad 2.5$ & 2.10 .1 \\
\hline $\mathrm{M} 2 \mathrm{~S}\left({ }^{13} \mathrm{C}\right)-\mathrm{S} 2 \mathrm{M}\left({ }^{1} \mathrm{H}\right)$ & $59.9 \quad 3.2$ & $2.5 \quad 0.2$ \\
\hline $\mathrm{M} 2 \mathrm{~S}\left({ }^{1} \mathrm{H}\right)-\mathrm{S} 2 \mathrm{M}\left({ }^{13} \mathrm{C}\right)$ & $61.6 \quad 2.0$ & $2.7 \quad 0.2$ \\
\hline
\end{tabular}

$\mathcal{H}_{J}^{\prime}$ respect the local symmetry of the four-spin system, while the terms $H_{Z}^{\prime}$ and $H_{J}^{\prime}$ contain the small symmetry-breaking terms induced by the remote asymmetric ester groups. In the experiments described here, the asymmetric term $\mathcal{H}_{\text {coh }}^{\prime}$ is exploited during the M2S and S2M sequences to access the state $\Delta_{\mathrm{gu}}$. However, during the evolution interval $\tau_{\mathrm{ev}}$, the effect of $\mathcal{H}_{\text {coh }}^{\prime}$ is suppressed by the applied RF field.

Spin permutation operators denoted $\left(i_{1} i_{2} \ldots i_{n}\right)$ may be defined, which lead to a cyclic permutation of $n$ spin labels when applied to a spin state or a spin operator. ${ }^{40}$ Different spin permutations may be multiplied. Examples are:

$$
\begin{gathered}
(12) I_{1 z} I_{3 z}(12)^{\dagger}=I_{2 z} I_{3 z} \\
(12)(34) I_{1 z} I_{3 z}(34)^{\dagger}(12)^{\dagger}=I_{2 z} I_{4 z}
\end{gathered}
$$

The symmetry group of the coherent Hamiltonian, denoted $\mathcal{G}_{\text {coh }}^{0}$, is defined here as the group of spin permutation operations which commutes with $\mathcal{H}_{\text {coh }}^{0}$.

In the current case, the group $\mathcal{G}_{\text {coh }}^{0}$ consists of just two operations, for both molecules:

$$
\mathcal{G}_{\mathrm{coh}}^{0}(\mathrm{AFD})=\mathcal{G}_{\mathrm{coh}}^{0}(\mathrm{AMD})=\{E,(14)(23)\}
$$

where $E$ is the identity. The presence of the permutation (14) indicates that spins $I_{1}$ and $I_{4}$ are chemically equivalent (as far as the idealized Hamiltonian $\mathcal{H}_{\text {coh }}^{0}$ is concerned). The fact that the permutation (14) does not appear in the group $\mathcal{G}_{\text {coh }}^{0}$ as an isolated element, but as a product with a different permutation (23), indicates that spins $I_{1}$ and $I_{4}$ are magnetically inequivalent.

The group $\mathcal{G}_{\text {coh }}^{0}$ has two irreducible representations denoted $A_{g}$ and $B_{u}$, with opposite parity under the operation (14)(23).

In the absence of any symmetry breaking interactions, the NMR spectral peaks are generated by transitions within the $A_{g}$ or $\mathrm{B}_{\mathrm{u}}$ manifolds.

4.1.1 Singlet-triplet product basis. We follow Warren et al. ${ }^{16}$ by considering a basis set which is a direct product of the singlet-triplet bases formed by homonuclear spin pairs:

$$
\mathbb{B}_{\mathrm{ST}}=\{\mathrm{ST}\}_{23} \otimes\{\mathrm{ST}\}_{14}
$$

A permutation $(i j)$ acting upon a ket belonging to $\{\mathrm{ST}\}_{i j}$ gives:

$$
(i j)\left|\psi_{i j}\right\rangle=p^{(i j)}\left|\psi_{i j}\right\rangle
$$

$\left|\psi_{i j}\right\rangle$ is said to be symmetric or gerade $(g)$ when $p^{(i j)}=+1$; antisymmetric or ungerade $(\mathrm{u})$ when $p^{(i j)}=-1$.

As different spin permutations may be multiplied, a fourspin state can be either symmetric $(\mathrm{g})$ or antisymmetric $(\mathrm{u})$
Table 3 The first column lists the components of the basis set $\mathbb{B}_{S T}$ in

\begin{tabular}{|c|c|c|c|c|}
\hline $\mathbb{B}_{\mathrm{ST}}$ & $p^{(23)}$ & $p^{(14)}$ & $p^{(14)(23)}$ & $M$ \\
\hline $\begin{array}{l}\left|\mathrm{T}_{1}{ }^{23} \mathrm{~T}_{1}{ }^{14}\right\rangle \\
\left|\mathrm{T}_{0}{ }^{23} \mathrm{~T}_{1}{ }^{14}\right\rangle \\
\left|\mathrm{T}_{1}{ }^{23} \mathrm{~T}_{0}{ }^{14}\right\rangle \\
\left|\mathrm{T}_{1}{ }^{23} \mathrm{~T}_{-1}{ }^{41}\right\rangle \\
\left|\mathrm{T}_{0}{ }^{23} \mathrm{~T}_{0}{ }^{14}\right\rangle \\
\left|\mathrm{T}_{-1}{ }^{23} \mathrm{~T}_{1}{ }^{41}\right\rangle \\
\left|\mathrm{T}_{0}{ }^{23} \mathrm{~T}_{-1} \mathrm{~T}_{-1}\right\rangle \\
\left|\mathrm{T}_{-1}{ }^{23} \mathrm{~T}_{0}{ }^{14}\right\rangle \\
\left|\mathrm{T}_{-1}{ }^{23} \mathrm{~T}_{-1}{ }^{14}\right\rangle\end{array}$ & $\begin{array}{l}\mathrm{g} \\
\mathrm{g} \\
\mathrm{g} \\
\mathrm{g} \\
\mathrm{g} \\
\mathrm{g} \\
\mathrm{g} \\
\mathrm{g} \\
\mathrm{g}\end{array}$ & $\begin{array}{l}\mathrm{g} \\
\mathrm{g} \\
\mathrm{g} \\
\mathrm{g} \\
\mathrm{g} \\
\mathrm{g} \\
\mathrm{g} \\
\mathrm{g} \\
\mathrm{g}\end{array}$ & $\begin{array}{l}\mathrm{g} \\
\mathrm{g} \\
\mathrm{g} \\
\mathrm{g} \\
\mathrm{g} \\
\mathrm{g} \\
\mathrm{g} \\
\mathrm{g} \\
\mathrm{g}\end{array}$ & $\begin{array}{r}2 \\
1 \\
1 \\
0 \\
0 \\
0 \\
-1 \\
-1 \\
-2\end{array}$ \\
\hline $\begin{array}{l}\left|\mathrm{S}_{0}{ }^{23} \mathrm{~S}_{0}{ }^{14}\right\rangle \\
\left|\mathrm{T}_{1}{ }^{23} \mathrm{~S}_{0}{ }^{14}\right\rangle \\
\left|\mathrm{T}_{0}{ }^{23} \mathrm{~S}_{0}{ }^{14}\right\rangle \\
\left|\mathrm{T}_{-1}{ }^{23} \mathrm{~S}_{0}{ }^{14}\right\rangle \\
\left|\mathrm{S}_{0}{ }^{23} \mathrm{~T}_{1}{ }^{14}\right\rangle \\
\left|\mathrm{S}_{0}{ }^{23} \mathrm{~T}_{0}{ }^{14}\right\rangle \\
\left|\mathrm{S}_{0}{ }^{23} \mathrm{~T}_{-1}{ }^{14}\right\rangle\end{array}$ & $\begin{array}{l}\mathrm{u} \\
\mathrm{g} \\
\mathrm{g} \\
\mathrm{g} \\
\mathrm{u} \\
\mathrm{u} \\
\mathrm{u}\end{array}$ & $\begin{array}{l}\mathrm{u} \\
\mathrm{u} \\
\mathrm{u} \\
\mathrm{u} \\
\mathrm{g} \\
\mathrm{g} \\
\mathrm{g}\end{array}$ & $\begin{array}{l}\mathrm{g} \\
\mathrm{u} \\
\mathrm{u} \\
\mathrm{u} \\
\mathrm{u} \\
\mathrm{u} \\
\mathrm{u}\end{array}$ & $\begin{array}{r}0 \\
1 \\
0 \\
-1 \\
1 \\
0 \\
-1\end{array}$ \\
\hline
\end{tabular}
eqn (11) used for describing both ${ }^{13} \mathrm{C}_{2}$-AFD, ${ }^{13} \mathrm{C}_{2}$-AMD. Each ket is classified according to its parity under exchange of the two ${ }^{13} \mathrm{C}$ nuclei, exchange of the two ${ }^{1} \mathrm{H}$ nuclei, and simultaneous exchange of both homonuclear pairs. The last column indicates the total magnetic quantum number

under the homonuclear spin permutation $(i j)(k l)$, that is $p^{(i j)(k l)}=$ $+1($ for $g)$ or $p^{(i j)(k l)}=-1$ (for $u$ ).

States belonging to $\mathbb{B}_{\mathrm{ST}}$ can be classified (see Table 2) according to the magnetic quantum number $M$ and their parities under the homonuclear spin permutations:

$$
\begin{aligned}
\left.\left(I_{1 z}+I_{2 z}+I_{3 z}+I_{4 z}\right)\left(\left|\psi_{14}\right\rangle \otimes\left|\psi_{23}\right\rangle\right)=M\left(\left|\psi_{14} \otimes\right| \psi_{23}\right\rangle\right) & \\
(14)\left(\left|\psi_{14}\right\rangle \otimes\left|\psi_{23}\right\rangle\right) & \left.=p^{(14)}\left(\left|\psi_{14} \otimes\right| \psi_{23}\right\rangle\right) \\
(23)\left(\left|\psi_{14}\right\rangle \otimes\left|\psi_{23}\right\rangle\right) & \left.=p^{(23)}\left(\left|\psi_{14} \otimes\right| \psi_{23}\right\rangle\right) \\
(14)(23)\left(\left|\psi_{14}\right\rangle \otimes\left|\psi_{23}\right\rangle\right) & =p^{(14)} p^{(23)}\left(\left|\psi_{14}\right\rangle \otimes\left|\psi_{23}\right\rangle\right) \\
& =p^{(14)(23)}\left(\left|\psi_{14}\right\rangle \otimes\left|\psi_{23}\right\rangle\right)
\end{aligned}
$$

The 16 states reported in Table 3 form an orthonormal basis set and are classified according to their symmetry with respect to the permutation (14)(23) into irreducible representations $A_{g}$ and $B_{u}$ :

$$
\Gamma_{\text {spin }}=10 \mathrm{~A}_{\mathrm{g}} \oplus 6 \mathrm{~B}_{\mathrm{u}}
$$

\subsection{Symmetry of the fluctuating hamiltonian}

Consider now the fluctuating spin interactions which cause relaxation. An example of such interactions is given by the dipole-dipole interaction between the nuclei, which may be written as follows:

$$
\mathcal{H}_{\mathrm{DD}}(t)=\sum_{\{i, j\}} \sum_{m=-2}^{+2} \sqrt{6} b_{i j} D_{0 m}{ }^{2}\left(\Omega_{\mathrm{PL}}^{i j}(t)\right) T_{2 m}^{i j}
$$

where the dipole-dipole coupling constant between a pair of nuclei is $b_{i j}=-\left(\mu_{0} / 4 \pi\right) \gamma_{i} \gamma_{j} \hbar r_{i j}{ }^{-3}, D^{2}$ is a second-rank Wigner rotation matrix, $\Omega_{\mathrm{PL}}^{i j}$ represents the set of three Euler angles defining the orientation of the internuclear vector between $I_{j}$ and $I_{k}$ in the laboratory frame, and $T_{2}^{i j}$ is a second-rank irreducible spherical tensor.

At an arbitrary time point $t$, molecular vibrations distort the molecular geometry away from its equilibrium configuration, 
breaking the geometrical symmetry. Hence, in general, the instantaneous value of $\mathcal{H}_{\mathrm{DD}}(t)$ (and other fluctuating terms) does not display symmetry. However, since rapid vibrations are usually too fast to cause significant NMR relaxation, the relevant spin Hamiltonian for relaxation purposes may be locally averaged over molecular vibrations (typically on the sub-picosecond timescale), denoted here $\left\langle\mathcal{H}_{\mathrm{DD}}(t)\right\rangle_{\mathrm{vib}}$. This vibrationally averaged spin Hamiltonian reflects the geometrical symmetry of the equilibrium molecular structure, and displays the corresponding spin permutation symmetry.

In general, $\left\langle\mathcal{H}_{\mathrm{DD}}(t)\right\rangle_{\text {vib }}$ may be calculated from eqn (15) by using nuclear coordinates from the equilibrium molecular geometry, but with small adjustments to the interaction strengths caused by vibrational averaging.

As previously done for $\mathcal{H}_{\text {coh }}$ we will consider in this section the nuclear spin permutations that are symmetry operations for $\left\langle\mathcal{H}_{\mathrm{DD}}(t)\right\rangle_{\text {vib }}$ in both molecular systems.

In the case of ${ }^{13} \mathrm{C}_{2}-\mathrm{AFD}$, the permutation (14)(23) is a symmetry operation for the vibrationally-averaged dipoledipole spin Hamiltonian because of the inversion geometry of the local molecular structure. The double permutation always exchanges pairs of nuclei with parallel internuclear vectors, so that the spatial interaction tensors are identical for all molecular orientations. Similar considerations apply for other interaction terms such as the chemical shift anisotropy.

The group of the vibrationally-averaged fluctuating Hamiltonian is therefore given by

$$
\mathcal{G}_{\text {fluc }}^{0}(\mathrm{AFD})=\{E,(14)(23)\}
$$

where $\mathcal{G}_{\text {fluc }}^{0}$ contains the group of spin permutation operators that commute with $\left\langle\mathcal{H}_{\text {fluc }}\right\rangle_{\text {vib }}$. In the case of the cis isomer, the equilibrium geometry is not sufficient to impose symmetry on the vibrationally averaged spin Hamiltonian. We therefore get a trivial symmetry group in this case,

$$
\mathcal{G}_{\text {fluc }}^{0}(\mathrm{AMD})=\{E\}
$$

Fig. 8 illustrates the symmetry properties of the vibrationally averaged intramolecular dipole-dipole interaction, in the two molecular systems. The figure shows matrix representations of $\left\langle H_{\mathrm{DD}}\right\rangle_{\text {vib }}$ in the basis $\mathbb{B}_{\mathrm{ST}}$. In the case of ${ }^{13} \mathrm{C}_{2}$-AFD (Fig. 8b), the matrix representation of $\left\langle H_{\mathrm{DD}}\right\rangle_{\mathrm{vib}}$ is block-diagonal in this basis, since the vibrationally averaged dipole-dipole Hamiltonian has the same symmetry as $\mathcal{H}_{\text {coh }}^{0}$ (eqn (10) and (16)). In the case of ${ }^{13} \mathrm{C}_{2}$-AMD (Fig. 8d), on the other hand, off-diagonal elements connecting different irreducible representations appear, since $\left\langle H_{\mathrm{DD}}\right\rangle_{\text {vib }}$ has a lower symmetry than $\mathcal{H}_{\text {coh }}^{0}$ (eqn (17)).

The sole consideration of the symmetry properties of the coherent hamiltonian (Fig. 8a and c) to infer the existence of LLS could be deceptive. The symmetry group for $\mathcal{H}_{\text {coh }}^{0}$ is identical for both ${ }^{13} \mathrm{C}_{2}$-AFD and ${ }^{13} \mathrm{C}_{2}$-AMD (eqn (10)) but a long-lived order is expected only in one case.

In general, if the molecular structure is rigid, and the equilibrium geometry of the local spin system is centrosymmetric, the group $\mathcal{G}_{\text {fluc }}^{0}$ has dimension 2 . If the molecule is rigid but noncentrosymmetric, $\mathcal{G}_{\text {fluc }}^{0}$ has dimension 1 . The symmetry properties
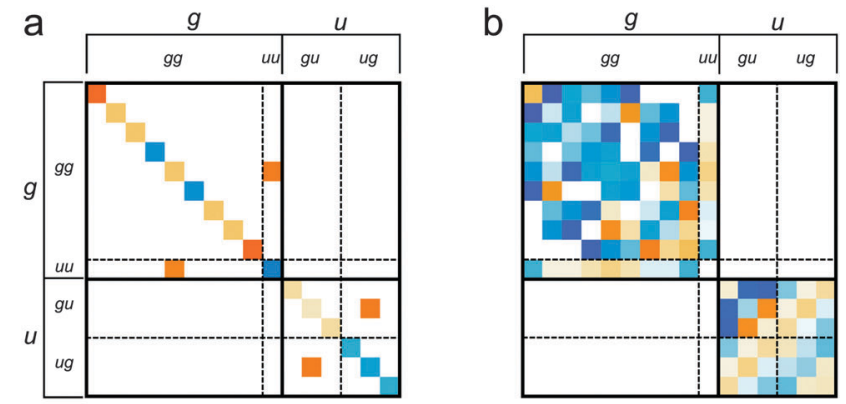

C

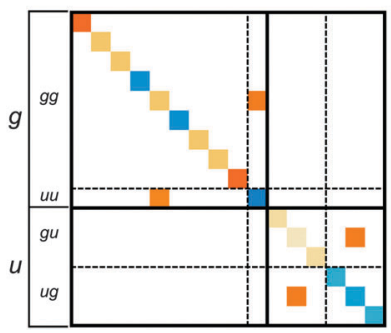

d

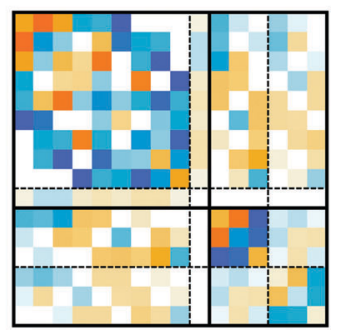

Fig. 8 Matrix plot representations of $\mathcal{H}_{\mathrm{coh}}^{0}$ and $\left\langle\mathcal{H}_{\mathrm{DD}}(t)\right\rangle_{\text {vib }}$ hamiltonians for ${ }^{13} \mathrm{C}_{2}$-AFD in panels (a), (b) and for ${ }^{13} \mathrm{C}_{2}$-AMD in panels (c) and (d) respectively. $\left\langle\mathcal{H}_{\mathrm{DD}}(t)\right\rangle_{\text {vib }}$ hamiltonian is block diagonal for ${ }^{13} \mathrm{C}_{2}-\mathrm{AFD}$ (panel (b)). The basis is $\mathbb{B}_{S T}$, and the ordering follows the convention adopted in Table 3.

of the coherent and fluctuating hamiltonians can be combined to derive the correct symmetry properties for the Liouvillian superoperator.

\subsection{Idealized Liouvillian group and long-lived states}

We define the idealized Liouvillian group of the spin system as the intersection of the groups $\mathcal{G}_{\text {coh }}^{0}$ and $\mathcal{G}_{\text {fluc }}^{0}$ :

$$
\mathcal{G}_{\mathcal{L}}^{0}=\mathcal{G}_{\text {coh }}^{0} \cap \mathcal{G}_{\text {fluc }}^{0}
$$

For the two molecules discussed here, we have:

$$
\begin{gathered}
\mathcal{G}_{\mathcal{L}}^{0}(\mathrm{AFD})=\{E,(14)(23)\} \\
\mathcal{G}_{\mathcal{L}}^{0}(\mathrm{AMD})=\{E\}
\end{gathered}
$$

The coherent and fluctuating Hamiltonians cannot induce transitions between states which belong to different irreducible representations of the group $\mathcal{G}_{\mathcal{L}}^{0}$. Hence the spin evolution must preserve a population difference between states belonging to different irreducible representations of $\mathcal{G}_{\mathcal{L}}^{0}$. Such a population difference is a constant of motion and does not evolve, providing that the realistic spin interactions conform exactly the idealized symmetry assumed in the theoretical model. In practice, deviations from ideal symmetry invariable cause slow relaxation, but the lifetime of such a state may nevertheless be long compared to the relaxation of most other states.

Suppose that $\mathcal{G}_{\mathcal{L}}^{0}$ has $n_{\mathcal{L}}$ irreducible representations. There are $n_{\mathcal{L}}$ combinations of populations which do not evolve, in the ideal case. However one of these constants of motion is the total spin system population, which is trivially conserved in all circumstances, for a closed system. 
Hence the number of non-trivial long-lived states $\left(N_{\text {LLS }}\right)$ is given by $n_{\mathcal{L}}-1$.

$$
N_{\text {LLS }}=n_{\mathcal{L}}-1
$$

In the case of ${ }^{13} \mathrm{C}_{2}$-AFD, which has a two-dimensional idealized Liouvillian group (eqn (19)), there is a LLS given by the difference in mean population of states with $\mathrm{A}_{\mathrm{g}}$ and $\mathrm{B}_{\mathrm{u}}$ symmetry:

$$
\Delta_{\mathrm{gu}}=\frac{1}{10} \sum_{i=1}^{10}\left|i, \mathrm{~A}_{\mathrm{g}}\right\rangle\left\langle i, \mathrm{~A}_{\mathrm{g}}\left|-\frac{1}{6} \sum_{i=1}^{6}\right| i, \mathrm{~B}_{\mathrm{u}}\right\rangle\left\langle i, \mathrm{~B}_{\mathrm{u}}\right|
$$

This corresponds to the following combination of spherical tensor operators:

$$
\Delta_{\mathrm{gu}}=T_{00}^{23}+T_{00}^{14}-4 \sqrt{3} T_{00} T_{00}^{14}
$$

where $T_{00}^{i j}=-3^{-1 / 2} \mathbf{I}_{i} \cdot \mathbf{I}_{j}$ is the rank-0 spherical tensor operator between nuclei $i$ and $j$. Eqn (23) highlights the invariance of the LLS under rotations of both spin ${ }^{1} \mathrm{H}$ and ${ }^{13} \mathrm{C}$ spin states, and can also be written as:

$$
\Delta_{\mathrm{gu}}=-\frac{1}{\sqrt{3}}\left(\mathbf{I}_{2} \cdot \mathbf{I}_{3}+\mathbf{I}_{1} \cdot \mathbf{I}_{4}-4\left(\mathbf{I}_{2} \cdot \mathbf{I}_{3}\right)\left(\mathbf{I}_{1} \cdot \mathbf{I}_{4}\right)\right)
$$

The operator $\Delta_{\text {gu }}$ is a LLS in the case of ${ }^{13} \mathrm{C}_{2}$-AFD, since the double spin permutation (14)(23) is an element of $\mathcal{G}_{\mathcal{L}}^{0}$ (AFD) (see eqn (19)). In the case of ${ }^{13} \mathrm{C}_{2}$-AMD, the idealized Liouvillian group is one-dimensional even in a regime of perfect chemical equivalence (see eqn (20)) and there is no long-lived state, except for the trivial mean of all spin state populations that is always a conserved quantity for an isolated spin system.

\subsection{Torsional angle dependence of the decay rate}

The dependence of the LLS on the equilibrium molecular geometry is now investigated. The arrangements of the four nuclear spins in ${ }^{13} \mathrm{C}_{2}$-AMD and ${ }^{13} \mathrm{C}_{2}$-AFD may be obtained from each another by rotating one of the heteronuclear ${ }^{13} \mathrm{CH}$ single bonds around the axis passing through the ${ }^{13} \mathrm{C}_{2}$ double bond.

The standard approach in LLS analysis consists in the diagonalization of the Liouvillian superoperator that can be set up to include all the relevant relaxation mechanisms. We will restrict our considerations to the relaxation induced by the intra-molecular dipole-dipole interaction (eqn (25)) for simplicity. When the dimension of the Hilbert space is $N$ the Liouvillian superoperator has a set of $N^{2}$ eigenvalues-eigenoperators pairs $\left\{L_{\mathrm{q}}, Q_{\mathrm{q}}\right\}$. In general $\hat{\mathcal{L}}_{\mathrm{DD}}$ is not hermitian so the eigenvalues may be complex.

$$
\begin{aligned}
& \hat{\mathcal{L}}_{\mathrm{DD}} Q_{\mathrm{q}}=L_{\mathrm{q}} Q_{\mathrm{q}} \\
& L_{\mathrm{q}}=-\lambda_{\mathrm{q}}+i \omega_{\mathrm{q}}
\end{aligned}
$$

Eqn (25) is the Liouville-von Neumann equation. Eigenoperators $Q_{\mathrm{q}}$ with complex eigenvalues correspond to coherences oscillating at frequency $\omega_{\mathrm{q}}$ and decaying to equilibrium with a rate constant $\lambda_{\mathrm{q}}$. Eigenoperators $Q_{\mathrm{q}}$ with real eigenvalues $\left(\omega_{\mathrm{q}}=0\right)$ correspond to spin state populations decaying with a rate $\lambda_{\mathrm{q}}$. The eigenvalue $\lambda_{\mathrm{q}}=0$ corresponds to the identity operator, corresponding to the conservation of the sum of all spin-state populations.
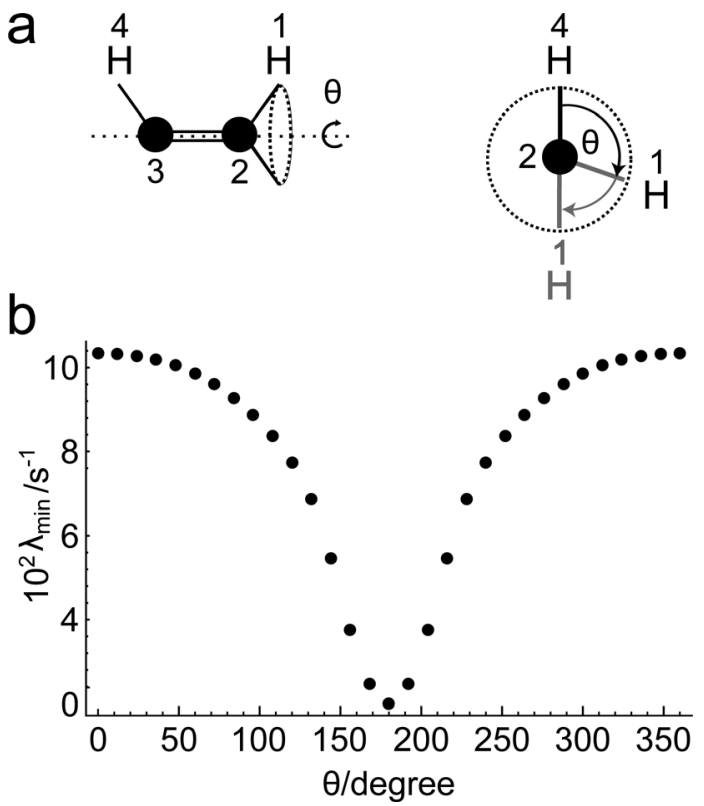

Fig. 9 (a) The geometric configurations obtainable by rotation of the single ${ }^{13} \mathrm{CH}$ bond through the ${ }^{13} \mathrm{C}_{2}$ double bond axis. The front view and the through ${ }^{13} \mathrm{C}_{2}$ double bond view are given. $\theta=0^{\circ}$ and $\theta=180^{\circ}$ correspond to equilibrium spin systems configurations of ${ }^{13} C_{2}$-AMD and ${ }^{13} C_{2}$-AFD respectively. (b) The smallest non-zero decay rate constant $\lambda_{\min }$ plotted against torsional angle $\theta$, obtained by numerical analysis of the Liouvillian superoperator. Only dipole-dipole relaxation is included, with a rotational correlation time of $\tau_{\mathrm{C}}=15 \mathrm{ps}$. A zero value, signing the existence of a infinitely LLS, is present only when $\theta=180^{\circ}$ : the geometric configuration of ${ }^{13} \mathrm{C}_{2}-\mathrm{AFD}$.

Numerical simulation performed with SpinDynamica $^{39}$ in Fig. 9b shows the dependence of the smallest non-zero rate constant $\lambda_{\text {min }}$ on the torsional angle $\theta$. It was assumed the $J$ coupling parameters to be independent of the torsional angle and equal to those reported in Table 1 for ${ }^{13} \mathrm{C}_{2}$-AFD. This approximation appears to be reasonable given the small difference in parameters as obtained by fitting the 1D-NMR spectra and listed in Table 1. The relaxation superoperator takes into account all dipole-dipole couplings in the 4-spin system and assumes rigid molecular geometry and isotropic molecular tumbling with a correlation time $\tau_{\mathrm{C}}=15 \mathrm{ps}$. As expected, a long-lived state with infinite relaxation time is predicted only when $\theta=180^{\circ}$, which is the geometric equilibrium configuration for ${ }^{13} \mathrm{C}_{2}$-AFD.

\subsection{Symmetry breaking}

Since applied fields are symmetric for all spins, the LLS described by eqn (22) can only be accessed by breaking the idealized Liouvillian symmetry $\mathcal{G}_{\mathcal{L}}^{0}$. In the current case, the symmetry-breaking occurs naturally through the term $\mathcal{H}_{\text {coh }}^{\prime}$, which is associated with the asymmetric ester substituents. The true symmetry group of the coherent Hamiltonian is therefore given by $\mathcal{G}_{\mathrm{coh}}=\{E\}$, instead of the idealized group $\mathcal{G}_{\text {coh }}^{0}$ in eqn (10). The true Liouvillian group is given by

$$
\mathcal{G}_{\mathcal{L}}=\mathcal{G}_{\text {coh }} \cap \mathcal{G}_{\text {fluc }}
$$

which in the current case is simply $\mathcal{G}_{\mathcal{L}}=\{E\}$ for both molecular systems. In the case of ${ }^{13} \mathrm{C}_{2}$-AFD, the symmetry-breaking 
perturbation generates terms connecting the irreducible representations of $\mathcal{G}_{\mathcal{L}}^{0}$, allowing experimental access to the LLS, through pulse sequences such as M2S, S2M, and relatives. ${ }^{8,9,20}$ In the study described here, the symmetry-breaking perturbation induced by the asymmetric ester substituents is large enough to provide experimental access to the LLS, but sufficiently small that a theoretical description based on the idealized Liouvillian group $\mathcal{G}_{\mathcal{L}}^{0}$ provides a good approximation.

Following the notation used throughout the script where ${ }^{1} \mathrm{H}$ nuclei are labelled 1 and 4 , and ${ }^{13} \mathrm{C}$ nuclei are labelled 2 and 3 , the theory developed could be also applied to the molecular AA'XX' 4-spin systems discussed for example in ref. 17, 18 and 25-27. The coherent spin Hamiltonian displays near-magneticequivalence, and the central spin pair is sufficiently remote from other participating spins that the symmetry group $\{E,(14),(23),(14)(23)\}$ is a reasonable approximation for both the coherent and the fluctuating Hamiltonians. This group has four irreducible representations, leading to three nontrivial long-lived states. Two of these may be accessed without breaking the chemical equivalence.

\section{Conclusions}

We have demonstrated the existence of a long-lived nuclear spin state in a multiple spin system, far from the usual conditions of near magnetic equivalence. A state of this kind is only supported by molecules with centrosymmetric local molecular geometry, such as fumarate. A group theoretical description of the conditions leading to long-lived states in multiple spin systems has been given. This theoretical approach is likely to be useful for understanding a variety of related problems, such as long-lived states in chemically equivalent spin systems ${ }^{16-19,26,27}$ and longlived states in rapidly rotating methyl groups. ${ }^{31}$

The existence of long-lived states in fumarate derivatives may also have practical relevance to hyperpolarized NMR studies of fumarate metabolism, in the context of in vivo cancer detection. ${ }^{41,42}$ We are currently exploring the possibility of generating the longlived population imbalance between the $\mathrm{A}_{\mathrm{g}}$ and $\mathrm{B}_{\mathrm{u}}$ manifolds directly through solid-state dynamic nuclear polarization (DNP), as has been demonstrated for singlet order in spin-pair systems. ${ }^{43}$

\section{Acknowledgements}

This research was funded by EPSRC (UK) and the European Research Council. We are grateful to Salvatore Mamone, JeanNicolas Dumez, Michael C. D. Tayler and Kevin Brindle for discussions and suggestions, and to Ole G. Johannessen for instrumental support.

\section{References}

1 M. Carravetta, O. Johannessen and M. Levitt, Phys. Rev. Lett., 2004, 92, 153003.

2 M. Carravetta and M. H. Levitt, J. Am. Chem. Soc., 2004, 126, 6228-6229.
3 M. Carravetta and M. H. Levitt, J. Chem. Phys., 2005, 122, 214505.

4 R. Sarkar, P. R. Vasos and G. Bodenhausen, J. Am. Chem. Soc., 2007, 129, 328-334.

5 G. Pileio and M. H. Levitt, J. Magn. Reson., 2007, 187, 141-145.

6 G. Pileio, M. Carravetta, E. Hughes and M. H. Levitt, J. Am. Chem. Soc., 2008, 130, 12582-12583.

7 M. C. D. Tayler, S. Marie, A. Ganesan and M. H. Levitt, J. Am. Chem. Soc., 2010, 132, 8225-8227.

8 G. Pileio, M. Carravetta and M. H. Levitt, Proc. Natl. Acad. Sci. U. S. A., 2010, 107, 17135-17139.

9 M. C. D. Tayler and M. H. Levitt, Phys. Chem. Chem. Phys., 2011, 13, 5556-5560.

10 M. H. Levitt, Annu. Rev. Phys. Chem., 2012, 63, 89-105.

11 G. Pileio, J. T. Hill-Cousins, S. Mitchell, I. Kuprov, L. J. Brown, R. C. D. Brown and M. H. Levitt, J. Am. Chem. Soc., 2012, 134, 17494-17497.

12 G. Pileio, S. Bowen, C. Laustsen, M. C. D. Tayler, J. T. HillCousins, L. J. Brown, R. C. D. Brown, J. H. Ardenkjaer-Larsen and M. H. Levitt, J. Am. Chem. Soc., 2013, 135, 5084-5088.

13 J.-N. Dumez, J. T. Hill-Cousins, R. C. D. Brown and G. Pileio, J. Magn. Reson., 2014, 246, 27-30.

14 A. K. Grant and E. Vinogradov, J. Magn. Reson., 2008, 193, 177-190.

15 W. S. Warren, E. Jenista, R. T. Branca and X. Chen, Science, 2009, 323, 1711-1714.

16 Y. Feng, R. M. Davis and W. S. Warren, Nat. Phys., 2012, 8, 831-837.

17 Y. Feng, T. Theis, T.-L. Wu, K. Claytor and W. S. Warren, J. Chem. Phys., 2014, 141, 134307.

18 K. Claytor, T. Theis, Y. Feng and W. Warren, J. Magn. Reson., 2014, 239, 81-86.

19 K. Claytor, T. Theis, Y. Feng, J. Yu, D. Gooden and W. S. Warren, J. Am. Chem. Soc., 2014, 136, 15118-15121.

20 S. J. DeVience, R. L. Walsworth and M. S. Rosen, Phys. Rev. Lett., 2013, 111, 173002.

21 N. Salvi, R. Buratto, A. Bornet, S. Ulzega, I. Rentero Rebollo, A. Angelini, C. Heinis and G. Bodenhausen, J. Am. Chem. Soc., 2012, 134, 11076-11079.

22 R. Buratto, D. Mammoli, E. Chiarparin, G. Williams and G. Bodenhausen, Angew. Chem., Int. Ed., 2014, 53, 11376-11380.

23 P. R. Vasos, A. Comment, R. Sarkar, P. Ahuja, S. Jannin, J.-P. Ansermet, J. a. Konter, P. Hautle, B. van den Brandt and G. Bodenhausen, Proc. Natl. Acad. Sci. U. S. A., 2009, 106, 18469-18473.

24 P. Ahuja, R. Sarkar, S. Jannin, P. R. Vasos and G. Bodenhausen, Chem. Commun., 2010, 46, 8192-8194.

25 L. Buljubasich, M. B. Franzoni, H. W. Spiess and K. Münnemann, J. Magn. Reson., 2012, 219, 33-40.

26 M. B. Franzoni, L. Buljubasich, H. W. Spiess and K. Münnemann, J. Am. Chem. Soc., 2012, 134, 10393-10396. 27 Y. Zhang, P. C. Soon, A. Jerschow and J. W. Canary, Angew. Chem., Int. Ed., 2014, 53, 3396-3399.

28 T. Theis, M. Truong, A. M. Coffey, E. Y. Chekmenev and W. S. Warren, J. Magn. Reson., 2014, 248C, 23-26. 
29 M. Emondts, M. P. Ledbetter, S. Pustelny, T. Theis, B. Patton, J. W. Blanchard, M. C. Butler, D. Budker and A. Pines, Phys. Rev. Lett., 2014, 112, 077601.

30 H. J. Hogben, P. J. Hore and I. Kuprov, J. Magn. Reson., 2011, 211, 217-220.

31 B. Meier, J.-N. Dumez, G. Stevanato, J. T. Hill-Cousins, S. S. Roy, P. H kansson, S. Mamone, R. C. D. Brown, G. Pileio and M. H. Levitt, J. Am. Chem. Soc., 2013, 135, 18746-18749.

32 M. H. Levitt, Spin dynamics. Basics of Nuclear Magnetic Resonance, 2001.

33 G. Pileio and M. H. Levitt, J. Chem. Phys., 2009, 130, 214501.

34 G. Pileio, M. Carravetta and M. H. Levitt, Proc. Natl. Acad. Sci. U. S. A., 2010, 107, 17135-17139.

35 T. Theis, Y. Feng, T.-l. Wu and W. S. Warren, J. Chem. Phys., 2014, 140, 014201.
36 H. J. Hogben, M. Krzystyniak, G. T. P. Charnock, P. J. Hore and I. Kuprov, J. Magn. Reson., 2011, 208, 179-194.

37 I. Kuprov, J. Magn. Reson., 2011, 209, 31-38.

38 A. Shaka, J. Keeler and R. Freeman, J. Magn. Reson., 1983, 53, 313-340.

39 M. H. Levitt, Spin Dynamica, 2013.

40 P. Bunker and P. Jensen, Molecular Symmetry and Spectroscopy, Academic Press, London, 2 edn, 2006, p. 721.

41 M. Karlsson, A. Gisselsson, S. K. Nelson, T. H. Witney, S. E. Bohndiek, G. Hansson, T. Peitersen, M. H. Lerche and K. M. Brindle, Proc. Natl. Acad. Sci. U. S. A., 2009, 106, 19801-19806.

42 K. M. Brindle, S. E. Bohndiek, F. a. Gallagher and M. I. Kettunen, Magn. Reson. Med., 2011, 66, 505-519.

43 M. C. D. Tayler, I. Marco-Rius, M. I. Kettunen, K. M. Brindle, M. H. Levitt and G. Pileio, J. Am. Chem. Soc., 2012, 134, 7668-7671. 